\title{
A geriatric patient with spinal cord injury without radiographic abnormality: outcomes and causes
}

\author{
Sevtap Acer $\mathbb{D}^{1} \cdot$ Ebru Karaca Umay ${ }^{1} \cdot$ Fatma Nazlı $^{1} \cdot$ Ugur Onur Kasman $^{2} \cdot$ İbrahim Gündoğdu $\cdot$ Aytül Çakcı $^{1}$
}

Received: 18 September 2017 / Revised: 05 February 2018 / Accepted: 5 February 2018

(c) International Spinal Cord Society 2018

\begin{abstract}
Introduction Although the prognosis of spinal cord injury without radiographic evidence of abnormality (SCIWORA) depends on the severity of the initial neurological damage, most patients with American Spinal Injury Association impairment scale grade D are expected to recover fully.

Case presentation An 85-year-old patient with SCIWORA and urinary incontinence, who did not produce the expected response to rehabilitation, displayed the central, peripheral, and autonomic nervous system findings together. Conventional radiography, computed tomography, and even magnetic resonance imaging were unable to explain this complicated neurological condition thoroughly. More in-depth research into the patient's history revealed some sequelae left by urinary surgery and chemotherapy.

Discussion Comorbidities in geriatric SCIWORA have severe effects on both etiology and prognosis. Furthermore, incontinence in SCIWORA is an essential condition that has not been addressed until now and may play a role in prognosis.
\end{abstract}

\section{Introduction}

Spinal cord injury without radiographic abnormality (SCIWORA) is a term used to define a clinical condition in which damage to the spinal cord or column cannot be seen with conventional radiography or computed tomography (CT), although magnetic resonance imaging (MRI) facilitates proper diagnosis [1]. Neurological signs range from transient paresthesia to tetraplegia. It is diagnosed by physical examination and radiographic imaging. Although this disease is most commonly found in pediatric age patients, it can also be seen, albeit rarely, in adults $[1,2]$.

The outcomes of neurological deficit recovery depend on many factors, including age, initial International Standards for Neurological Classification of Spinal Cord Injury (ISNCSCI) grade, MRI findings, and education level.

Sevtap Acer

sevtap.acer@marmara.edu.tr

1 Department of Physical Medicine and Rehabilitation, Diskapi Yildirim Beyazit Training and Research Hospital, Sehit Omer Halisdemir Street, Altindag, Ankara, Turkey

2 Department of Orthopaedics and Traumatology, Diskapi Yildirim Beyazit Training and Research Hospital, Sehit Omer Halisdemir Street, Altindag, Ankara, Turkey
Causes such as comorbid diseases affect the outcome. Pathogenesis, incidence, and outcome vary among different age groups because of anatomical and biomechanical differences [1, 3-7]. Previous studies have limited data concerning the clinical prognosis of SCIWORA because of the rarity of the disease in adults, especially in geriatric patients. Furthermore, urinary incontinence in SCIWORA and its follow-up is not well known. In this case study, the difficulty of diagnosis due to premorbid comorbidities, as well as some factors that can affect the prognosis in a geriatric patient with SCIWORA syndrome are discussed.

\section{Case presentation}

An 85-year-old male patient, who previously had independent ambulation, had a fall that induced low-energy trauma and was admitted to the emergency room. His cervical, thoracic, and lumber conventional radiographs did not reveal any fracture or dislocation. A cervical CT showed only mild degenerative changes in the cervical vertebral bodies. His motor and sensory levels were determined to be level C3 and grade D, according to ISNCSCI. He was diagnosed with cervical SCIWORA syndrome. In addition to degeneration, the T2-weighted MRI showed a uniform hyperintense intramedullary signal with $2 \mathrm{~cm}$ sagittal length in the spinal cord 
at the level of the C5 vertebra and the T1-weighted images showed isointense cord suggestive of spinal cord edema.

The patient with upper and lower extremity weakness and sensory symptoms was referred to our rehabilitation clinic at the third week of the event. During the physical examination performed at the beginning of his rehabilitation program, his cranial nerves were intact, the upper and lower extremity proximal muscle strengths were $3 / 5$, and the distal muscle strengths were $2 / 5$. His hand functions were weak, including grasping (Frenchay Arm Test score: 1/5). In addition to the altered sensory perception below $\mathrm{C} 3$, a protective and deep sense loss was observed at the hands and feet predominantly. Distal deep tendon reflexes were hypoactive, superficial reflexes were normal, and pathological reflexes were negative. Bulbocavernosus reflexes, superficial and deep anal sensations, and voluntary anal contraction existed. The urological clinical assessment and bladder diaries suggested an urge type of urinary incontinence; however, the cystometry and pressure flow studies indicated decreased bladder capacity with no overactivity. Respiration, swallowing, and bowel functions were normal. Although multidirectional rehabilitation was being performed, the etiology that did not fit the first motor neuron damage was investigated.

The patient's background revealed that he has a history of type 2 diabetes mellitus, which is regulated with oral antidiabetics, as well as colon and prostate adenocarcinoma, which was cured with resection and chemotherapy 2 years ago, and transurethral resection of the prostate (TURP). He had disclosed that he used platinum-based chemotherapeutics due to colon cancer, which led to numbness in the hands and feet. An electrophysiological examination showed moderate sensorimotor axonal degeneration in the upper and lower extremities and bilateral severe carpal tunnel syndrome. We also learned that his incontinence had started after the TURP operation and increased after the fall.

After a 3-week multidirectional rehabilitation program, significant improvement was recorded in the proximal upper and the lower extremity muscle strength, with no substantial change in the distal muscle strength. Despite conservative treatments including lifestyle advice, pelvic floor muscle exercises, bladder control strategies, electrical stimulation therapy, and a pharmacological option (tolterodine), there was no clinically significant change in the bladder function. He was unable to reach independent ambulation in the sixth week after the injury. He was discharged on his own request and was reported to have continued the therapy at home.

\section{Discussion}

SCIWORA is a syndrome that is often seen in childhood and is characterized by spinal cord injury (SCI) occurring after trauma with no evidence of trauma by direct radiography or CT [7, 8]. It constitutes $13.7-38 \%$ of SCI in the pediatric age group and in $<10 \%$ of adult SCI [8]. Preexisting spinal stenosis and intervertebral disc disease increase the risk of more significant damage after simpler injuries in adults $[1,2,9,10]$.

In studies concerning etiology, Liu et al. investigated 59 (2\%) of 2964 adult SCI patients [10]. Traffic accidents, sports injuries, and falls were reported as etiologic agents. A total of 40 patients had injuries at the thoracic level, and 19 patients had injuries at the cervical level. Of the 32 patients with complete injury, central cord syndrome was noted in seven cases. Thirty-nine patients did not undergo MRI due to complete neurological recovery in 24 hours. Guo et al. reported that falls $(52.2 \%)$ and vehicular injury $(28.6 \%)$ were the most common causes of injury [11]. The most predominantly affected level was found to be $\mathrm{C} 4 / 5(48.7 \%)$, followed by $\mathrm{C} 5 / 6(30.5 \%)$ and $\mathrm{C} 3 / 4$ (12.8\%), respectively. In this case study, the neurological ISNCSCI level was C3 and MRI level was C5. The etiology of cervical SCIWORA is a low-energy fall while walking. The fact that he fell on a flat surface and did not regain independence in ambulation might be related to preexisting nerve damage.

Although the diagnosis of SCIWORA using MRI is controversial, positive MRI findings are present in most patients. Among patients with MRI, in the study of Liu et al., $45 \%$ had degenerative and spondylotic changes, 12 had cord contusion, 7 had cord edema, 2 had cord hemorrhage, and 4 had ligamentum flavum hypertrophy [10]. Gupta et al. evaluated 15 SCIWORA patients with MRI and found that intervertebral disc prolapse was present in 6 of the patients, intramedullary hematoma/contusion in 4 , cord edema in 4, and multiple disc prolapses and intramedullary hematoma was present in 1 of the patients [9]. Boese and Lechler reviewed 44 studies that reported 1132 cases with MRI imaging, which they classified into four types: Type I (no MRI finding), Type IIa (extraneural), Type IIb (intraneural), and Type IIc (combined extraneural and intraneural MRI abnormalities) [1]. Our patient's MRI finding was classified as Type IIb, according to Boese and Lechler.

The therapeutic approaches to SCIWORA are highly variable, and given the lack of prospective trials, there are no evidence-based recommendations in this regard [1]. It is still based on individual decisions and includes conservative and operative options. In one of the cohorts of Boese, patients were treated with physiotherapy and/or neurorehabilitation facilities, except for those classified as Type IIc who underwent surgical decompression, and the mean improvement of American Spinal Injury Association Impairment Scale (AIS, ISNCSCI) grade was 1.4 units in the patients with Type IIb lesions [3]. Our patient, who had a Type IIb lesion and an AIS grade of D, had not undergone surgery. 
Many studies state the relationship between prognosis and the extensity of the cord damage $[1,4,7,9,10,12]$. Although the patients with severe neurological impairment have poor prognosis, moderate and mild cases may have better outcomes. Liu et al. reported $61 \%$ of patients had complete recovery, $39 \%$ had partial improvement at the time of discharge, and two patients continued to have neurological deficit 6 months after the discharge [10]. Additionally, no relation between MRI findings and prognosis was noted. In contrast, some studies show that a cord transection or a major hemorrhage seen by MRI are associated with worse outcomes than an edema or the absence of any findings [7, 9, 12]. A retrospective study by MartinezPerez demonstrates that lesion length is a powerful predictor of the result, and the injury of soft tissue plays a role in the ability to recover in adult SCIWORA [4]. Boese end Lechler reported that imaging findings reveal the best prognosis for Type I, followed by Type IIa, Type IIb, and Type IIc, respectively. Neurologic impairment at admission and follow-up showed highly significant differences in outcomes between patients with different imaging findings [1]. In our case, despite negative prognostic factors such as advanced age, significant improvement in the patient's proximal muscle strength was noted. However, he was still unable to ambulate. Failure to achieve full recovery and ambulation level was most likely due to the premorbid peripheral neurological deficits and comorbidities of the patient.

Peripheral neuropathy may occur as a side effect of various cancer drugs and as a complication of diabetes mellitus. Peripheral neuropathy due to cancer chemotherapy is reported to have a frequency between 3 and 38\% [13]. Research shows that these agents may decrease the amplitude of action potential (particularly in sensory neurons), decrease transmission speed, cause pain, or frequent cause axonal and/or demyelinating types of neuropathy [13-15]. Sensory symptoms usually start in the lower extremities bilaterally and typically make glove-and-sock-type polyneuropathy with hand involvement in time. Atonic bladder, impotence, and orthostatic hypotension may also occur in cancer chemotherapy-induced autonomic neuropathy. Concomitantly, diabetes or alcohol use may increase the incidence or severity [13-15]. The axonal polyneuropathy detected in our case was associated with the use of platinum-based chemotherapeutics and a background of diabetes.

There may be many reasons for the incontinence in our patient. The clinical finding of the sudden urinary leaking and the laboratory finding of the decreased bladder capacity with no overactivity may be related to coexistence of central and peripheral nervous system disorder [16, 17]. Motor and sensory loss in the lower extremities may improve quickly, whereas bladder and colon dysfunction can continue for a long time in children with SCIWORA [18]. However, we have not encountered any study concerning incontinence in adults with SCIWORA in the literature. Among the probable causes, urge incontinence is not a rare $(11 \%)$ disorder in the male population over age 65 [19]. The main mechanism of the urge incontinence is an overactive bladder; however, the cystometry and the pressure flow studies did not show overactivity in our patient. This may be explained with preexisting neuropathy. On the other hand, the patient's surgical history, especially TURP, seem to be the most likely cause. Although the transient incontinence after TURP is reported to be $30-40 \%$, it may be permanent rarely [20].

Advanced age, stroke, diabetes, poor general health, prostate cancer, radical prostatectomy, TURP, and radiotherapy have been reported to increase the risk of urinary incontinence, and of these, radical prostatectomy increases the risk the most [19]. For the treatment of urinary incontinence, a similar approach as used in adults is recommended in a healthy geriatric population; however, if it is accompanied by comorbidities, drug use, or functional or cognitive impairment, a more specific approach may be required $[16,17]$. We did not observe any improvement in the urinary continence symptoms in our patient. This may be related to the causes mentioned above, such as advance age, diabetes, cancer, and surgery history. However, readers should consider whether 6 weeks after the SCIWORA is enough time or not for the improvement in bladder symptoms due to the lack of literature.

In conclusion, although this syndrome usually has a good prognosis, geriatric age, comorbidities, and the presence of positive findings on an MRI can lead to an insufficient recovery. Considering the differences in the mechanisms of injury, preexisting degenerative disorders, and comorbidities found in adults and the geriatric population, further studies are needed in the geriatric population with SCIWORA.

Acknowledgements There are no funding, grants, or equipment provided for the project from any source. There are not any financial benefits to the authors. This abstract and manuscript have not been presented anywhere before.

\section{Compliance with ethical standards}

Conflict of interest The authors declare that they have no conflict of interest.

\section{References}

1. Boese CK, Lechler P. Spinal cord injury without radiologic abnormalities in adults: a systematic review. J Trauma Acute Care Surg. 2013;75:320-30. 
2. Izma MK, Zulkharnain I, Ramli B, Muhamad AR, Harwant S. Spinal cord injury without radiological abnormality (SCIWORA). Med J Malays. 2003;58:105-10.

3. Boese CK, Muller D, Broer R, Eysel P, Krischek B, Lehmann HC, et al. Spinal cord injury without radiographic abnormality (SCIWORA) in adults: MRI type predicts early neurologic outcome. Spinal Cord. 2016;54:878-83.

4. Martinez-Perez R, Munarriz PM, Paredes I, Cotrina J, Lagares A. Cervical spinal cord injury without computed tomography evidence of trauma in adults: magnetic resonance imaging prognostic factors. World Neurosurg. 2017;99:192-9.

5. Mohanty SP, Bhat NS, Singh KA, Bhushan M. Cervical spinal cord injuries without radiographic evidence of trauma: a prospective study. Spinal Cord. 2013;51:815-8.

6. Neva MH, Roeder CP, Felder U, Kiener B, Meier W, Perler M, et al. Neurological outcome, working capacity and prognostic factors of patients with SCIWORA. Spinal Cord. 2012;50:78-80.

7. Pang D, Pollack IF. Spinal cord injury without radiographic abnormality in children--the SCIWORA syndrome. J Trauma. 1989;29:654-64.

8. Brown RL, Brunn MA, Garcia VF. Cervical spine injuries in children: a review of 103 patients treated consecutively at a level 1 pediatric trauma center. J Pediatr Surg. 2001;36:1107-14.

9. Gupta SK, Rajeev K, Khosla VK, Sharma BS, Paramjit, Mathuriya SN, et al. Spinal cord injury without radiographic abnormality in adults. Spinal Cord. 1999;37:726-9.

10. Liu Q, Liu Q, Zhao J, Yu H, Ma X, Wang L. Early MRI finding in adult spinal cord injury without radiologic abnormalities does not correlate with the neurological outcome: a retrospective study. Spinal Cord. 2015;53:750-3.
11. Guo H, Liu J, Qi X, Ning G, Zhang H, Li X, et al. Epidemiological characteristics of adult SCIWORA in Tianjin, China: a preliminary study. Eur Spine J. 2012;21:165-71.

12. Grabb PA, Pang D. Magnetic resonance imaging in the evaluation of spinal cord injury without radiographic abnormality in children. Neurosurgery. 1994;35:406-14. discussion 14.

13. Jaggi AS, Singh N. Mechanisms in cancer-chemotherapeutic drugs-induced peripheralneuropathy. Toxicology. 2012;291:1-9.

14. Han Y, Smith MT. Pathobiology of cancer chemotherapy-induced peripheral neuropathy (CIPN). Front Pharmacol. 2013;4:156.

15. Sioka C, Kyritsis AP. Central and peripheral nervous system toxicity of common chemotherapeutic agents. Cancer Chemother Pharmacol. 2009;63:761-7.

16. Abrams P, Andersson KE, Birder L, Brubaker L, Cardozo L, Chapple $\mathrm{C}$, et al. Fourth International Consultation on Incontinence Recommendations of the International Scientific Committee: evaluation and treatment of urinary incontinence, pelvic organ prolapse, and fecal incontinence. Neurourol Urodyn. 2010;29:213-40.

17. Bardsley A. An overview of urinary incontinence. Br J Nurs. 2016;25:S14-S21.

18. Bauer SB. Neurogenic bladder: etiology and assessment. Pediatr Nephrol. 2008;23:541-51.

19. Shamliyan TA, Wyman JF, Ping R, Wilt TJ, Kane RL. Male urinary incontinence: prevalence, risk factors, and preventive interventions. Rev Urol. 2009;11:145-65.

20. Rassweiler J, Teber D, Kuntz R, Hofmann R. Complications of transurethral resection of the prostate (TURP)--incidence, management, and prevention. Eur Urol. 2006;50:969-79. 